\title{
六胜肽片段的液相合成及组装研究
}

\author{
张 腾 $a, b$ 宋 卫 ${ }^{b} \quad$ 韩 涁 $^{a}$ 刘 林 ${ }^{b} \quad$ 冯凌云 $^{b}$ \\ 赵金礼 ${ }^{b}$ 刘建利*,a \\ ( ${ }^{a}$ 西北大学生命科学学院 西部资源生物与现代生物技术省部共建教育部重点实验室 西安 710069) \\ ( ${ }^{b}$ 西安华澳丽康活性蛋白与多肽工程中心 西安 710054)
}

\begin{abstract}
摘要 六胜肽是一种药妆肽, 序列为 Ac-Glu-Glu-Met-Gln-Arg-Arg- $\mathrm{NH}_{2}$, 是相对安全的类肉毒素活性物质. 拟用液相方 法分别合成片段 A [Ac-Glu(OtBu)-Glu(OtBu)-Met-OH]和片段 B [H-Gln(Trt)-Arg-Arg-OH], 然后将两个片段在固相载体 上进行组装，经切割、纯化得到六胜肽产品，纯度大于 $98 \%$, 片段组装的收率为 $65 \%$, 并对组装反应的条件进行分析和 讨论. 该方法结合了固相和液相合成的优点, 是一种成本低廉、收率较高和周期较短的六胜肽合成方法. 关键词 六胜肽; 片段肽; 合成
\end{abstract}

\section{Synthesis of Hexapeptide by Liquid-Phase Fragments Coupling Strategy}

\author{
Zhang, Teng ${ }^{a, b} \quad$ Song, $\mathrm{Wei}^{b} \quad \mathrm{Han}^{\mathrm{B}} \mathrm{Bin}^{a} \quad \mathrm{Liu} \mathrm{Lin}^{b} \quad$ Feng, Lingyun ${ }^{b}$ \\ Zhao, Jinli $^{b} \quad$ Liu, Jianli ${ }^{*, a}$ \\ ( ${ }^{a}$ Key Laboratory of Resource Biology and Biotechnology in Western China, Ministry of Education, \\ College of Life Science, Northwest University, Xi'an 710069) \\ ( ${ }^{b}$ Active Protein \& Polypeptide Engineering Center of Xi'an Hua Ao Li Kang, Xi'an 710054)
}

\begin{abstract}
The hexapeptide (Ac-EEMQRR- $\mathrm{NH}_{2}$ ) is a non-toxic cosmeceutical which can mimic the function of botulinum neurotoxins. This paper reports a solid-phase synthesis of hexapeptide by employing " $A+B$ " fragments coupling strategy while fragment A $[\mathrm{Ac}-\mathrm{Glu}(\mathrm{OtBu})-\mathrm{Glu}(\mathrm{OtBu})-\mathrm{Met}-\mathrm{OH}]$ and $\mathrm{B}[\mathrm{H}-\mathrm{G} \ln (\mathrm{Trt})-\mathrm{Arg}-\mathrm{Arg}-\mathrm{OH}]$ were prepared by classical solution methods. The final product (coupling yield $>65 \%$, purity $>98 \%$ ) was obtained by cleavage cocktail treatment and HPLC purification. The influencing factors of coupling condition were discussed. In conclusion, this strategy is a relatively quickly, efficient and high yield method for the synthesis of hexapeptide by combining the advantages of solid phase and liquid phase synthesis.

Keywords hexapeptide; peptide fragments; synthesis
\end{abstract}

多肽制备最早是通过有机液相反应完成的，首先将 羧基活化, 然后与下一个氨基酸反应生成酰胺, 依次重 复制得目标肽. 该方法的特点是, 对于短肽的合成具有 规模化生产的可能, 但对长肽的制备大多不太理想. 固 相合成方法的出现有效解决了长肽的合成问题 ${ }^{[1]}$, 其特 点是将肽链连接在固相载体上, 通过过量反应物提高反 应收率，同时反复洗涤就能除去杂质，大大节省了反应
时间. 然而，固相载体的交换当量非常有限，价格又较 贵, 一般只能进行小批量合成, 不能满足实际需求 ${ }^{[2]}$. 因此，根据不同肽链的结构特点，选择合适的合成方法 是非常必要的. 通常, 将固相和液相方法结合，进行分 段合成是较为理想的多肽制备方法 ${ }^{[3]}$, 即先通过液相法 合成片段肽，再用固相方法对片段肽进行组装. 我们要 报道基于这种思路合成六胜肽的一种方法.

\footnotetext{
*E-mail: jlliu@nwu.edu.cn

Received March 20, 2014; revised May 12, 2014; published online June 9, 2014.

Project supported by the National Natural Science Foundation of China (Nos. 20872118, 30070905), the Key Laboratory Fund of Shaanxi Province of China (Nos. 2010JS097, 11JS090, 12JS110), the Foundation of the Education Department of Shaanxi Province (No. 08jk477) and the Technology Innovation Fund of Xi'an City (No. CX13120)

国家自然科学基金(Nos. 20872118, 30070905)、陕西省重点实验室基金(Nos. 2010JS097, 11JS090, 12JS110)、陕西省教育厅基金(No. 08jk477)和西安市 技术创新(No. CX13120)资助项目.
} 
六胜肽是一种药妆肽, 序列为 Ac-Glu-Glu-MetGln-Arg-Arg- $\mathrm{NH}_{2}$ (图 1), 是由突触蛋白 SNAP-25 的氮端 (AA12 17) 衍生而来的六个氨基酸肽链, 能与 SNARE 三元复合物相互作用, 抑制嘹铬细胞中受 $\mathrm{Ca}^{2+}$ 调控的 儿茶酚胺释放 ${ }^{[4]}$, 从而阻止神经-肌肉间的乙酰胆碱传 导, 引起肌肉麻㾇. 六胜肽是肉毒素类的安全替代品, 与之活性相似, 具有膜渗透性, 有效避免了潜在的毒副 作用 ${ }^{[5]}$. 由于能够阻断神经肌肉间的信息传导, 避免肌 肉过度收缩, 减少了动态纹的发生, 同时还能有效重组 胶原弹力, 增加弹力蛋白活性, 使脸部线条放松 ${ }^{[6]}$, 因 此, 多用于化妆品内作为抗皱成分 ${ }^{[7]}$.

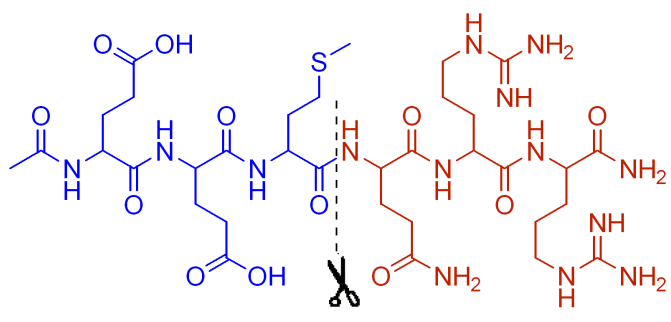

Fragment $\mathrm{A}$

Fragment B

图 1 六胜肽结构

Figure 1 Structures of Hexapeptide

目前，有关六胜肽的制备均采用经典固相法完 成 ${ }^{[4 \mathrm{a}, 5]}$, 然而该方法不够灵活, 导致成本很高, 收率较 小. 为解决这一问题, 本文采用液相法分别合成片段 $\mathrm{A}$ [Ac-Glu(OtBu)-Glu(OtBu)-Met-OH] (Scheme 1)和片段 B [H-Gln(Trt)-Arg-Arg-OH] (Scheme 2), 再将两个片段用 固相法组装 (Scheme 3), 制得六胜肽产品. 该方法既有 液相法降低成本、易于放大的优点, 又有固相法易于纯
化、操作简单的特点，是一种相对理想的六胜肽合成方 法.

\section{1 结果与讨论}

\section{1 片段肽的合成}

肽键的形成主要通过活化酯的氨解完成，使用 $\mathrm{HOSu}$ 活化能有效抑制产物消旋，并减少 $N$-酰基脲等副 产物的生成. 但对于化合物 B-2, 由于精氨酸的侧链较 长以及胍基的存在, 需使用 $\mathrm{HONB}$ 作活化剂, $\mathrm{N}, \mathrm{N}$-二甲 基甲酰胺(DMF)为溶剂才能活化, 因为 HONB 自身的空 间位阻较小，且 DMF 的促增溶作用使反应在均相条件 下进行，有利于充分反应. 在氨解反应过程中，无论有 机碱还是无机碱，均具有相同效果的催化作用。一般, 控制用量不低于游离氨基酸的摩尔量( $1 \sim 1.5$ equiv.), 使反应体系在碱性环境中, 便能有效促进氨解反应进 行 ${ }^{[8]}$ (图 2).
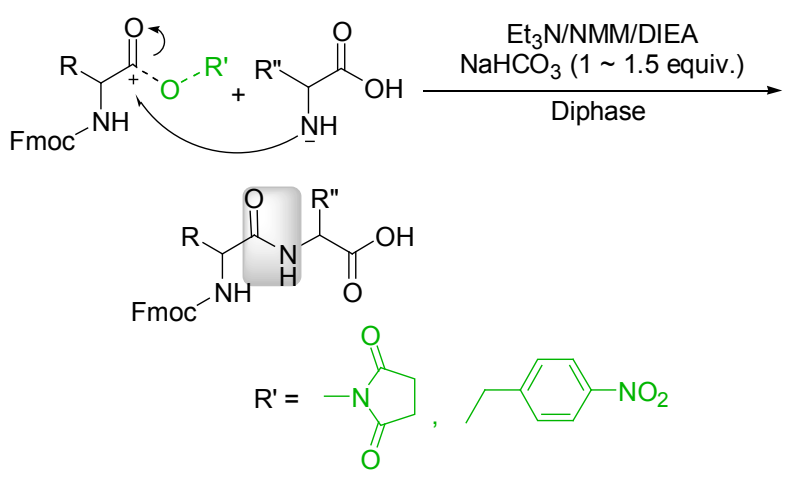

图 2 活化酯的碱催化氨解

Figure 2 Base catalysis of the aminolysis of activated ester

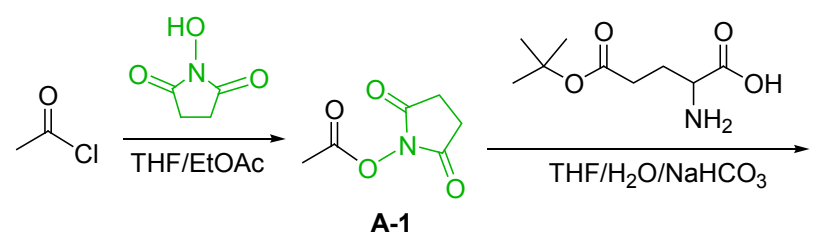<smiles>CC(C)(C)OC(=O)CCCC(N)C(=O)O</smiles>

$\mathrm{THF} / \mathrm{H}_{2} \mathrm{O} / \mathrm{NaHCO}_{3}$<smiles>CC(=O)NC(CCC(=O)OC(C)(C)C)C(=O)NC(CCC(=O)OC(C)(C)C)C(=O)O</smiles>

A-4<smiles>COC(=O)NOC(=O)C(N)CCSC</smiles><smiles>CC(=O)N[C@@H](CCCC(=O)OC(C)(C)C)C(=O)O</smiles>
A-2

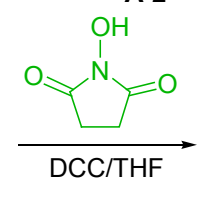

A-3<smiles>CC(C)C(=O)NC(CCC(=O)OC(C)(C)C)C(=O)NC(=O)ON1C(=O)CCC1=O</smiles><smiles>CC(=O)NC(CCC(=O)OC(C)(C)C)C(=O)NC(CCC(=O)OC(C)(C)C)C(=O)O</smiles>

A-5

Scheme 1 
<smiles>CCNC(=O)CCC(NC(F)F)C(=O)O</smiles>

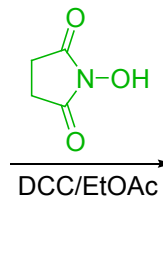<smiles>CCCCC(=O)NC(=O)CCC(NC(F)F)C(=O)ON1C(=O)CCC1=O</smiles><smiles>N=C(N)NCCCC(N)C(=O)O</smiles><smiles>O=[N+]([O-])c1ccc(CO)cc1</smiles><smiles>CCCNC(=O)CCC(NC(=O)NCC(F)F)C(=O)NC(CCCNC(=N)N)C(=O)OCc1ccc([N+](=O)[O-])cc1</smiles><smiles>N=C(N)NCCCC(N)C(=O)O</smiles><smiles>CCCNC(=O)CCC(NC(=O)NCCCNC(=N)N)C(=O)NC(CCCNC(=N)N)C(=O)NC(CCCNC(=O)O)C(=O)O</smiles>

Scheme 2

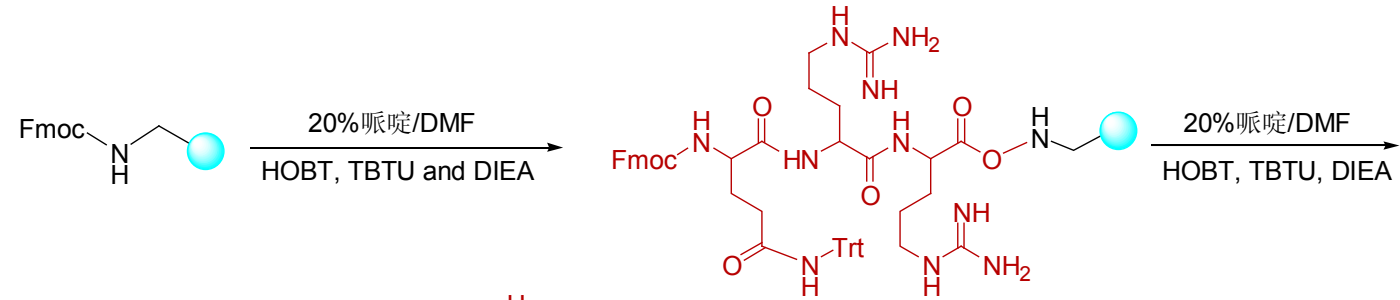<smiles>CCCCNC(=O)CCC(NC(=O)C(CCSC)NC(=O)C(CCC(=O)N[I+])NC(=O)C(CCC(=O)OC(C)(C)C)NC(=O)OC(C)(C)C)C(=O)NC(CCCNC(=N)N)C(=O)ONC</smiles>
$83: 5: 4: 3: 5$<smiles>CSCCC(NC(=O)C(CCC(=O)O)NC(=O)C(CCC(=O)O)NC(C)=O)C(=O)NC(CCC(N)=O)C(=O)NC(CCCNC(=N)N)C(=O)NC(CCCNC(=N)N)C(N)=O</smiles><smiles>CNC(c1ccc(OC=NCc2ccc(C)cc2)cc1)c1ccc(OC)cc1OC</smiles>

Scheme 3

表 1 液相合成与固相组装反应条件的比较

Table 1 Different reaction conditions between liquid-phase and solid-phase synthesis

\begin{tabular}{cccccc}
\hline Phase & Coupling agent & Catalyst & \multicolumn{1}{c}{ Solvent } & Cat./equiv. & Temperature $/{ }^{\circ} \mathrm{C}$ \\
\hline Liquid-phase & $\mathrm{HOSu} / \mathrm{HONB}$ & $\mathrm{DCC}$ & $\mathrm{THF}, \mathrm{EtOAc}, \mathrm{DMF} / \mathrm{H}_{2} \mathrm{O}$ & $1.0 \sim 1.5$ & $<0$ \\
Solid-phase & $\mathrm{HOBt}, \mathrm{TBTU}$ & $\mathrm{DIEA}$ & $\mathrm{DMF} / \mathrm{DMSO} / \mathrm{NMP}$ & $3 \sim 4$ & r.t. \\
\hline
\end{tabular}

肽类化合物有其结构特殊性，据此可对各片段及中 间体进行方便的纯化. 如 A-2 有较强的疏水性质, 可用 酸化沉淀法析出; 而 A-4 中存在两个叔丁酯保护基, 亲
脂性较强，可以用混合溶剂结晶法纯化; A-6 和 B-4 均是 三肽片段, 可用乙醚超声处理纯化, 这可能是借助了乙 醚分子的静电作用; B-2 由于引入了精氨酸, 亲脂性较 
差, 可用乙酸乙酯打浆处理; 过量的游离氨基酸均可用 水林洗掉，因此后处理较为简便.

\section{2 片段组装的条件}

起初, 我们尝试通过活化酯的方法链接两个片段 肽, 但发现二者的缩合效率非常低, 很难纯化. 原因可 能有两方面: 首先, 片段 $\mathrm{A}$ 是一个三肽片段, 受肽链自 身影响, 其活化酯的活性要比单个氨基酸或二肽的活化 酯偏低; 另外, 片段 B 中含有的 Arg-Arg 是肽链合成中 的困难序列 ${ }^{[9,10]}$, 其中两个胍基的多重氢键作用使分子 间发生紧密聚集, 溶剂化程度降低, 溶解性变差, 导致 缩合效率降低. 因此, 改用固相片段缩合的方法进行连 接(表 1), 一方面, 增加相应活化剂(HOBT、TBTU)和催 化剂(DIEA)的用量(3 4 equiv.); 另一方面, 改用等体 积比的混合溶液 (DMF/DMSO/NMP) 作溶剂. 这样不仅 改善了活化剂的活性, 还由于混合溶剂中 $\mathrm{C}=\mathrm{O}$ 与肽链 中 $\mathrm{N}-\mathrm{H}$ 间的氢键作用 ${ }^{[11]}$, 减弱了肽链间聚集的倾向, 增加了片段的溶解性和树脂的溶胀性, 使反应试剂分 子、可溶的构件分子在较短的时间内能充分自由接触, 从而提高片段缩合的效率.

\section{3 反应时间对缩合效率的影响}

片段肽因其结构变化和分子量增大, 理化性质完全 不同于单个氨基酸, 一般会使反应变缓慢, 缩合率降低, 因此, 除考虑缩合剂、催化剂、溶剂等因素外, 还很有 必要延长反应时间. 采用 $0.05 \mathrm{~g} / \mathrm{mL}$ 的狮三酮/乙醇溶液 加热显色的方法, 考察不同反应时间(分别为 $0.5,1,1.5$, $2,2.5,3,4,5,6,7 \mathrm{~h}$ )树脂载体的变色情况, 即正检时阴 性反应占树脂取样量的体积百分比, 来评价反应进程.

由图 3 可以看出, 随着反应时间的增加, 片段 $\mathrm{B}$ (B-4)与树脂的连接率在 $3 \mathrm{~h}$ 之前逐渐升高, 反应进行到 $5 \mathrm{~h}$ 时, 缩合率最高, 约为 $93 \%$, 继续反应, 提高的程度 逐渐趋于平缓. 在图 4 中, 片段 A (A-6)与片段 B 的连接 率在 $2.5 \mathrm{~h}$ 之前迅速升高, 约 $3.5 \mathrm{~h}$ 便达到最佳收率. 原 因可能是片段 $\mathrm{A}$ 较片段 $\mathrm{B}$ 的溶解性能更好, 且空间位阻 小, 活化氨解反应更易进行, 从而表现出较好的连接率.

\section{4 六胜肽质谱和液相分析图}

从图 5 可以看出, 采用梯度洗脱方式分析六胜肽产 品, 程序为 $5 \% \sim 65 \%, 25 \mathrm{~min}[\mathrm{~A}(0.1 \% \mathrm{TFA} / \mathrm{ACN}), \mathrm{B}$ $\left(0.1 \% \mathrm{TFA} / \mathrm{H}_{2} \mathrm{O}\right)$ ], 出峰时间为 $7.85 \mathrm{~min}$, 纯度 $98.38 \%$. 分子量为 888.9, 质谱中出现 $m / z: 889.5\left(\mathrm{M}^{+}, 100\right)$, $890.45\left[(\mathrm{M}+1)^{+}, 50\right], 445\left[(\mathrm{M}+1)^{+} / 2,40\right]$, 结合 $\mathrm{IR}$ 和 ${ }^{1} \mathrm{H}$ NMR 给出的信息可以确定结构.

\section{2 结论}

以液相活化酯的方法合成六胜肽中的两各片段, 然

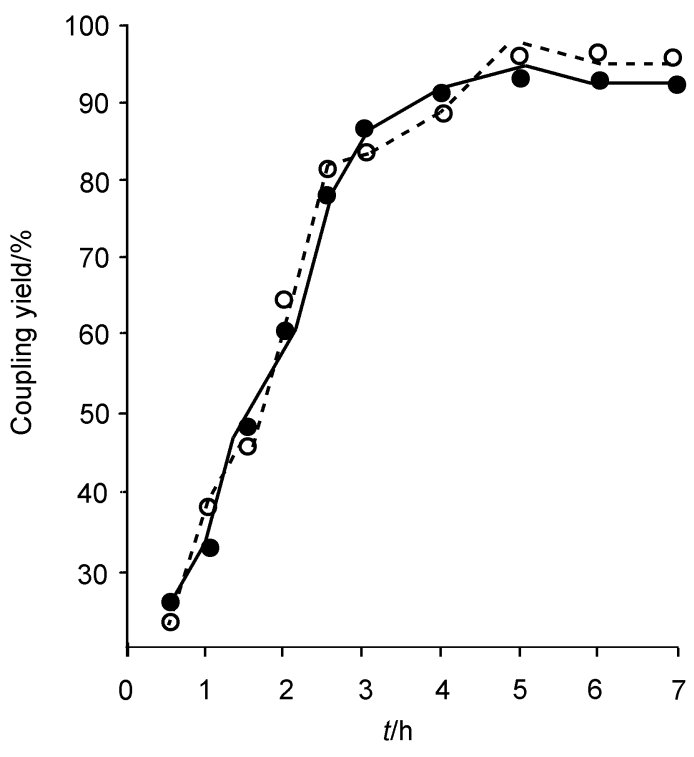

图 3 反应时间对片段 B 与树脂缩合效率的影响 Figure 3 Influence of reaction time on coupling yield between B-4 and resin

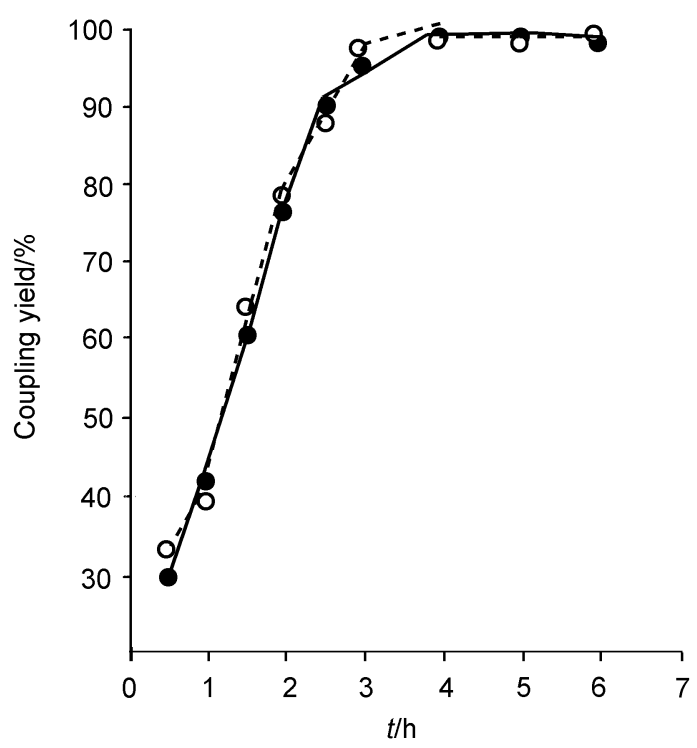

图 4 反应时间对片段 A 与片段 B 缩合效率的影响

Figure 4 Influence of reaction time on coupling yield between fragments $\mathrm{A}$ and $\mathrm{B}$

后在固相载体上进行连接, 最后经切割、纯化, 得到六 胜肽产品 $5.83 \mathrm{~g}$, 纯度大于 $98 \%$, 片段组装收率为 $65.53 \%$. 与经典固相法相比, 片段肽和目标多肽的纯度 和产率得到明显提高, 说明该方法是一种周期较短、成 本低廉和收率较高的合成方法, 为六胜肽及其它多肽在 液相合成方面的研究提供参考.

\section{3 实验部分}

\section{1 试剂与仪器}

X-5 显微熔点测定仪(未校准, 北京泰克仪器有限 


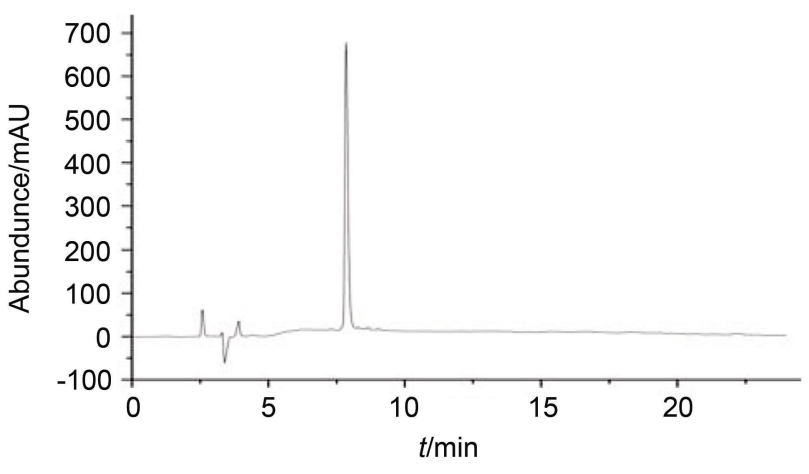

图 5 六胜肽质谱和色谱分析

Figure 5 HPLC-MS analysis of hexapeptide

公司); LTQ-XL 电喷雾电离质谱(美国 Thermo Finnigan 公司); Bruker Avance-400MHz 核磁共振仪(TMS 作内标, $\mathrm{D}_{2} \mathrm{O}$ 为溶剂, 瑞士 Bruker 公司); 分析高效液相色谱仪 (日立全自动 L2000); C18 分析色谱柱 $4.6 \mathrm{~mm} \times 250 \mathrm{~mm}$ (大连物理化学研究所); 制备高效液相色谱仪(创新通恒 LC3000); C18 制备色谱柱 $40.1 \mathrm{~mm} \times 450 \mathrm{~mm}$ (成都科普 生物有限公司); FT-IR (Spectrum Two)红外分光光度计 (美国 Perkin Elmer 公司); Autopol II 旋光仪(美国 Rudolph 公司); Rink Amide-MBHA Resin $(0.8 \sim 1.0$ $\mathrm{mmol} / \mathrm{g}$ )购于天津南开和成科技有限公司; 乙酰氯 $(\mathrm{CR}$ 级), 购于上海国药试剂有限公司; $N$-差基丁二酰亚胺 ( $\mathrm{HOSu}, \mathrm{CR}$ 级)购于渭南惠丰化工有限公司; 二环己基 碳二酰亚胺 (DCC, CR 级) 购于苏州天马有限公司; Fmoc-Gln(Trt)-OH 、 H-Glu(OtBu)-OH 、 H-Met-OH 、 $\mathrm{H}-\mathrm{Arg}-\mathrm{OH}$ 、1-差基苯并三氮唑 $(\mathrm{HOBt}, \mathrm{CR}$ 级)购于上海 吉尔化工有限公司; 其他试剂均为国产 $\mathrm{AR}$ 或 $\mathrm{CR}$ 级试 剂, 部分试剂使用前进行干燥或蒸馏处理. 缩写: HONB，4-硝基苯甲醇; TBTU，2-(1H-苯并三偶氮 $L-1$ 基)-1,1,3,3-四甲基腿四氟硼酸酯; DIEA, $N, N$-二异丙基 乙胺; EtOAc, 乙酸乙酯; $\mathrm{MOH}$, 甲醇; $\mathrm{PE}$, 石油醚; THF，四氢呋喃; DMF, $N, N$-二甲基甲酰胺; TFA, 三氟 乙酸; Phenol,苯酚; Tis, 三异丙基硅烷; DMSO, 二甲基 亚砜; NMP, $N$-甲基吡咯烷酮; $i$-PrOH, 异丙醇; $\mathrm{Et}_{3} \mathrm{~N}$, 三 乙胺; NMM, 氮甲基吗啉. 溶液配比均指体积.

\section{2 片段 $\mathrm{A}$ 的合成}

\subsection{1 合成 $\mathrm{AcOSu}(\mathbf{A}-1)$}

将 $11.5 \mathrm{~g}$ (100 mmol) HOSu 置于 $250 \mathrm{~mL}$ 三口瓶中, 依次加入 $100 \mathrm{~mL}$ 干燥的 THF 和 $19.9 \mathrm{~mL}(120 \mathrm{mmol})$ DIEA, 插上温度计和干燥器, 冰盐浴条件下不断搅拌, 并缓慢滴加 $7.9 \mathrm{~mL}(110 \mathrm{mmol}) \mathrm{AcCl}$, 滴完后撤去冰浴, 室温反应 $3 \mathrm{~h}$, 反应液不经处理直接用于下步反应.

\subsection{2 合成 $\mathrm{Ac}-\mathrm{Glu}(\mathrm{OtBu})-\mathrm{OH}(\mathbf{A}-2)$}

将 $22.4 \mathrm{~g}$ (110 mmol) H-Glu(OtBu)-OH 和 $16.8 \mathrm{~g}$
(200 mmol) $\mathrm{NaHCO}_{3}$ 溶于 $75 \mathrm{~mL}$ 蒸馏水中, 所得溶液滴 加到上步溶有 $\mathrm{AcOSu}$ 的 $\mathrm{THF}$ 溶液中, 持续摚拌, 反应 液逐渐澄清. TLC 监测反应, 约 $4 \sim 6 \mathrm{~h}$, 过滤固体不溶 物, 减压浓缩滤液, 用质量分数为 $20 \%$ 的柠檬酸水溶液 调节 $\mathrm{pH}$ 值至 $2 \sim 3$, 并不断搅拌, 逐渐析出大量固体, 冰箱中静置, 过滤, 用水淋洗滤饼, $40{ }^{\circ} \mathrm{C}$ 真空干燥, 得 到白色粉末状 $\mathrm{Ac}-\mathrm{Glu}(\mathrm{OtBu})-\mathrm{OH} 21.5 \mathrm{~g}$, 收率为 $87.7 \%$. m.p. $173.5 \sim 174.6{ }^{\circ} \mathrm{C}$, TLC: $R_{\mathrm{f}}=0.3, V($ EtOAc $):$ $V($ Hexane $): V(\mathrm{AcOH})=10: 10: 1$. HPLC: A $(0.1 \%$ TFA/ACN $), B\left(0.1 \% \mathrm{TFA} / \mathrm{H}_{2} \mathrm{O}\right), 30 \mathrm{~min} 10 \% \sim 90 \%$, $12.88 \mathrm{~min}$. 纯度 $92.8 \%$; ESI-MS m/z: $246\left(\mathrm{M}^{+}, 10\right), 268$ $\left(\mathrm{M}+\mathrm{Na}^{+}, 60\right), 189\left(\mathrm{M}^{+}-56,100\right), 171(30)$.

\subsection{3 合成 Ac-Glu(tBu)-COOSu (A-3)}

将 $21.25 \mathrm{~g}$ (86.7 mmol) Ac-Glu(OtBu)-OH 置于 500 $\mathrm{mL}$ 三口瓶中, 依次加入 $200 \mathrm{~mL}$ 干燥的 THF 和 $12 \mathrm{~g}$ (104 $\mathrm{mmol}) \mathrm{HOSu}$, 插上温度计和干燥器, 冰盐浴条件下不 断摚拌, 并缓慢滴加溶有 $23.25 \mathrm{~g}(112.8 \mathrm{mmol}) \mathrm{DCC}$ 的 THF $(50 \mathrm{~mL})$ 溶液, 滴完后撤去冰浴, TLC 监测反应至完 成后, 过滤除去固体不溶物, 滤液为溶有 $\mathrm{Ac}-\mathrm{Glu}(\mathrm{OtBu})-$ $\mathrm{COOSu}$ 的 THF 溶液.

\subsection{4 合成 $\mathrm{Ac}-\mathrm{Glu}(\mathrm{OtBu})-\mathrm{Glu}(\mathrm{OtBu})-\mathrm{OH}(\mathbf{A}-4)$}

将 $19.4 \mathrm{~g}(95.5 \mathrm{mmol}) \mathrm{H}-\mathrm{Glu}(\mathrm{OtBu})-\mathrm{OH}$ 和 $10.93 \mathrm{~g}$ (130 mmol) $\mathrm{NaHCO}_{3}$ 溶于 $200 \mathrm{~mL}$ 蒸馏水中, 所得溶液 滴加到上步溶有 Ac-Glu(OtBu)-COOSu 的 THF 溶液中, 持续摚拌, 反应液逐渐澄清. TLC 监测, 至反应完成, 过 滤固体不溶物, 减压浓缩滤液, 用质量分数为 $20 \%$ 的柠 檬酸水溶液调节 $\mathrm{pH}$ 值至 $2 \sim 3$, EtOAc 萃取 $(100 \mathrm{~mL} \times 1$, $50 \mathrm{~mL} \times 2)$, 并依次用水 $(50 \mathrm{~mL} \times 3)$ 、饱和食盐水 $(50$ $\mathrm{mL} \times 2$ ) 洗涤有机相, 无水硫酸钠干燥, 再加入 $3 \mathrm{~g}$ 活性 碳于 $40 \sim 45{ }^{\circ} \mathrm{C}$ 摚拌 10 $20 \mathrm{~min}$ ，趁热过滤，滤液减压浓 缩, 得到透明的糖浆状粘稠物(约 $35 \mathrm{~g}$ ). 用 EtOAc 与 PE 体积比为 $1.3: 1$ 的混合液重结晶, 析出固体, 过滤、干 燥, 得白色粉末状 Ac-Glu(OtBu)-Glu(OtBu)-OH $25 \mathrm{~g}$, 收率 67.0\%. m.p. $113.7 \sim 117.1{ }^{\circ} \mathrm{C}$, TLC: $R_{\mathrm{f}}=0.2$, $V($ EtOAc) $: V(\mathrm{Hex}): V(\mathrm{AcOH})=15: 5: 1$. HPLC: A $(0.1 \% \mathrm{TFA} / \mathrm{ACN}), \mathrm{B}\left(0.1 \% \mathrm{TFA} / \mathrm{H}_{2} \mathrm{O}\right), 30 \mathrm{~min} 10 \%$ $90 \%, 17.63 \mathrm{~min}$. 纯度 $86.7 \%$; ESI-MS $m / z$ : $431\left(\mathrm{M}^{+}, 60\right)$, $454\left(\mathrm{M}+\mathrm{Na}^{+}, 100\right)$.

\subsection{5 合成 Ac-Glu(OtBu)-Glu(OtBu)-COOSu (A-5)}

将 $24 \mathrm{~g}(55.9 \mathrm{mmol}) \mathrm{Ac}-\mathrm{Glu}(\mathrm{OtBu})-\mathrm{Glu}(\mathrm{OtBu})-\mathrm{OH}$ 置 于 $500 \mathrm{~mL}$ 三口瓶中, 依次加入 $170 \mathrm{~mL}$ 干燥的 THF 和 $7.72 \mathrm{~g}(67.3 \mathrm{mmol}) \mathrm{HOSu}$, 插上温度计和干燥器, 冰盐 浴条件下不断搅拌, 并缓慢滴加溶有 $15 \mathrm{~g}(72.4 \mathrm{~mol})$ DCC 的 THF $(40 \mathrm{~mL})$ 溶液, TLC 监测反应. 约 $6 \mathrm{~h}$ 后, 过 滤除去固体不溶物, 滤液为溶有 $\mathrm{Ac}-\mathrm{Glu}(\mathrm{OtBu})-$ 
$\mathrm{Glu}(\mathrm{OtBu})-\mathrm{COOSu}$ 的四氢呋喃溶液.

3.2.6 合成 Ac-Glu(OtBu)-Glu(OtBu)-Met-OH (A-6)

将 $9.98 \mathrm{~g}(66.9 \mathrm{mmol})$ H-Met-OH 和 $7.1 \mathrm{~g}(83.7$ $\mathrm{mmol}) \mathrm{NaHCO}_{3}$ 溶于 $120 \mathrm{~mL}$ 蒸馏水中, 所得溶液滴加到 上步溶有 Ac-Glu(OtBu)-Glu(OtBu)-COOSu 的 THF 溶液 中, 持续摚拌, TLC 监测反应. 约 $10 \sim 12 \mathrm{~h}$ 后, 过滤固体 不溶物, 减压浓缩滤液, 用质量分数为 $20 \%$ 的柠檬酸水 溶液调节 $\mathrm{pH}$ 值至 $2 \sim 3$, 用 EtOAc 萃取 $(70 \mathrm{~mL} \times 3)$, 并 依次用水 $(70 \mathrm{~mL} \times 2)$ 、饱和食盐水 $(70 \mathrm{~mL} \times 2)$ 洗涤有机 相, 无水硫酸钠干燥, 过滤, 滤液减压浓缩, 乙醚超声 处理 $(70 \mathrm{~mL} \times 2)$, 得到 $\mathrm{Ac}-\mathrm{Glu}(\mathrm{OtBu})-\mathrm{Glu}(\mathrm{OtBu})-\mathrm{Met}-$ $\mathrm{OH} 25.5 \mathrm{~g}$, 收率为 $81.2 \%$. m.p. $127.2 \sim 130.0{ }^{\circ} \mathrm{C}$, TLC: $R_{\mathrm{f}}=0.3, V(\mathrm{EtOAc}): V(\mathrm{Hex}): V(\mathrm{MeOH}): V(\mathrm{AcOH})=$ $10: 10: 1: 1$. HPLC: A $(0.1 \%$ TFA/ACN $)$, B $(0.1 \%$ TFA/ $\mathrm{H}_{2} \mathrm{O}$ ), $30 \mathrm{~min} 10 \% \sim 90 \%, 19.83 \mathrm{~min}$. 纯度 $96.2 \%$; ESI-MS $m / z$ : $562\left(\mathrm{M}^{+}, 100\right) ; 561\left(\mathrm{M}^{+}-1,80\right)$.

\section{3 片段 $\mathrm{B}$ 的合成}

\subsection{1 合成 Fmoc-Gln(Trt)-COOSu (B-1)}

将 $61 \mathrm{~g}$ (100 mmol) Fmoc-Gln(Trt)-OH 置于 $1000 \mathrm{~mL}$ 三口瓶中, 依次加入 $300 \mathrm{~mL}$ 干燥的 EtOAc 和 $13.8 \mathrm{~g}$ (120 mmol) $\mathrm{HOSu}$, 插上温度计和干燥器, 冰盐浴条件 下不断摚拌, 并缓慢滴加溶有 $26.8 \mathrm{~g}(100 \mathrm{mmol}) \mathrm{DCC}$ 的 EtOAc $(120 \mathrm{~mL})$ 溶液, TLC 监测反应. $4 \sim 5 \mathrm{~h}$ 后, 过滤除 去固体不溶物, 滤液悬蒸干, 不经处理直接用于下步反 应.

\subsection{2 合成 Fmoc-Gln(Trt)-Arg-OH (B-2)}

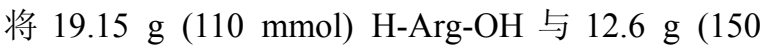
$\mathrm{mmol}) \mathrm{NaHCO}_{3}$ 溶于 $200 \mathrm{~mL}$ 蒸馏水中, 然后滴加到上步 溶有 Fmoc-Gln(Trt)-COOSu 的 THF 溶液中, 持续摚拌, TLC 监测反应. 约 $12 \mathrm{~h}$, 过滤, 减压浓缩滤液, 用质量 分数为 $20 \%$ 的柠檬酸水溶液调节 $\mathrm{pH}$ 值至 $2 \sim 3$, 用 EtOAc 萃取 $(100 \mathrm{~mL} \times 4)$, 并依次用水 $(100 \mathrm{~mL} \times 2)$ 、饱 和食盐水 $(100 \mathrm{~mL} \times 2)$ 洗涤有机相, 无水硫酸钠干燥, 过 滤, 滤液减压浓缩, 得类白色固体约 $69 \mathrm{~g}$. 用 $100 \mathrm{~mL}$ 乙 酸乙酯打浆处理, 得到 Fmoc-Gln(Trt)-Arg-OH 62.5g, 收 率为 $81.5 \%$. m.p. $161.1 \sim 164.3{ }^{\circ} \mathrm{C}$. TLC: $R_{\mathrm{f}}=0.3$, $V(\mathrm{EtOAc}): V(\mathrm{Hex}): V(\mathrm{MeOH}): V(\mathrm{AcOH})=10: 10:$ $1: 1$. HPLC: A $\left(0.1 \%\right.$ TFA/ACN), B $\left(0.1 \%\right.$ TFA $\left./ \mathrm{H}_{2} \mathrm{O}\right), 30$ $\min 10 \% \sim 90 \%, 23.31 \mathrm{~min}$. 纯度 95\%; ESI-MS m/z: 767 $\left(\mathrm{M}^{+}, 100\right)$.

\subsection{3 合成 Fmoc-Gln(Trt)-Arg-COONB (B-3)}

将 $60 \mathrm{~g}$ (78.3 mmol) Fmoc-Gln(Trt)-Arg-OH 置于 500 $\mathrm{mL}$ 三口瓶中, 依次加入 $140 \mathrm{~mL}$ 干燥的 DMF 和 $14.4 \mathrm{~g}$ (94 mmol) HONB, 插上温度计和干燥器, 冰盐浴条件
下不断搅拌, 并缓慢滴加溶有 $21 \mathrm{~g}(101.8 \mathrm{mmol}) \mathrm{DCC}$ 的 DMF $(30 \mathrm{~mL})$ 溶液, TLC 监测反应. 约 $8 \mathrm{~h}$ 后, 过滤除去 固体不溶物, 滤液为溶有 Fmoc-Gln(Trt)-Arg-COONB 的 DMF 溶液.

\subsection{4 合成 Fmoc-Gln(Trt)-Arg-Arg-OH (B-4)}

将 $15 \mathrm{~g}$ (86.15 mmol) H-Arg-OH 和 $9.87 \mathrm{~g}$ (117.45 $\mathrm{mmol}) \mathrm{NaHCO}_{3}$ 溶于 $80 \mathrm{~mL}$ 蒸馏水中, 所得溶液滴加到 上步溶有 Fmoc-Gln(Trt)-Arg-COONB 的 DMF 溶液中, 持续摚拌, $\mathrm{TLC}$ 监测反应. 约 $15 \sim 20 \mathrm{~h}$ 后, 用油泵减压 浓缩至 $100 \mathrm{~mL}$, 用质量分数为 $20 \%$ 的柠檬酸水溶液调 节 $\mathrm{pH}$ 值至 2 3, 追加水至体积 $300 \mathrm{~mL}$, 用 EtOAc 与 $\mathrm{MeOH}$ 体积比为 $4: 1$ 的混合液萃取 $(100 \mathrm{~mL} \times 3)$, 饱和 食盐水 $(70 \mathrm{~mL} \times 2)$ 洗涤有机相, 无水硫酸钠干燥, 过滤, 滤液减压浓缩, 得到粗品(约 $45 \mathrm{~g})$, 用乙醚 $(100 \mathrm{~mL} \times 2)$ 超声处理, 得到类白色固体 Fmoc-Gln(Trt)-Arg-Arg-OH $29.1 \mathrm{~g}$, 收率为 $40.26 \%$. m.p. $108 \sim 111.5{ }^{\circ} \mathrm{C}$, HPLC: A $(0.1 \% \mathrm{TFA} / \mathrm{ACN}), \mathrm{B}\left(0.1 \% \mathrm{TFA} / \mathrm{H}_{2} \mathrm{O}\right), 30 \mathrm{~min} 30 \%$ $90 \%, 17.93 \mathrm{~min}$. 纯度 $93.7 \%$; ESI-MS $m / z$ : $924\left(\mathrm{M}^{+}, 40\right)$, $923(80)$.

\section{4 片段的组装}

\subsection{1 合成 Fmoc-Gln(OTrt)-Arg-Arg-MBHA Resin}

反应器中加入 $10.0 \mathrm{~g}$ Rink Amide MBHA Resin 和 $100 \mathrm{~mL}$ DMF, 浸泡树脂 $30 \mathrm{~min}$, 使充分溶胀. 抽滤除去 DMF，加入 $80 \mathrm{~mL} 20 \%$ 的哌啶/DMF 溶液，反应 $10 \mathrm{~min}$, 抽滤, 再加入 $80 \mathrm{~mL} 20 \%$ 的哌啶/DMF 溶液, 反应 20 min, 抽滤, 分别用 $i-\mathrm{PrOH}(80 \mathrm{~mL} \times 2)$ 和 $\mathrm{DMF}(80$ $\mathrm{mL} \times 3$ )洗涤树脂. 然后向反应器中加入 $140 \mathrm{~mL}$ 混合液 $V(\mathrm{DMF}): V(\mathrm{DMSO}): V(\mathrm{NMP})=1: 1: 1,27.7 \mathrm{~g}$ FmocGln(Trt)-Arg-Arg, $4.06 \mathrm{~g}$ HOBT, $9.64 \mathrm{~g}$ TBTU 和 $4.97 \mathrm{~mL}$ DIEA, 氮气保护下室温反应, $0.05 \mathrm{~g} / \mathrm{mL}$ 狮三酮/乙醇溶 液监测树脂显色变化, 约 $5 \mathrm{~h}$, 抽滤, 分别用 $i$-PrOH (100 $\mathrm{mL} \times 2)$ 和混合液 $V(\mathrm{DMF}): V(\mathrm{DMSO}): V(\mathrm{NMP})=1:$ $1: 1(100 \mathrm{~mL} \times 2)$ 洗涤树脂, 抽滤, 得到 Fmoc-Gln(Trt)Arg-Arg-MBHA Resin.

3.4.2 合成 Ac-Glu(OtBu)-Glu(OtBu)-Met-Gln(Trt)Arg-Arg-MBHA Resin

在装有 Fmoc-Gln(Trt)-Arg-Arg-MBHA Resin 的反 应器中, 加入 $100 \mathrm{~mL}$ 混合液 $V$ (哌啶)：V(DMF)： $V(\mathrm{DMSO}): V(\mathrm{NMP})=1: 1: 1: 1$ 反应 $10 \mathrm{~min}$, 抽滤, 再加入 $100 \mathrm{~mL}$ 混合液 $V$ (哌啶)：V(DMF)：V(DMSO)： $V(\mathrm{NMP})=1: 1: 1: 1$, 反应 $20 \mathrm{~min}$, 抽滤, 分别用 $i$-PrOH $(100 \mathrm{~mL} \times 2)$ 和混合液 $V(\mathrm{DMF}): V(\mathrm{DMSO}):$ $V(\mathrm{NMP})=1: 1: 1(100 \mathrm{~mL} \times 2)$ 洗涤树脂. 然后向反应 器中加入 $140 \mathrm{~mL}$ 混合液 $V(\mathrm{DMF}): V(\mathrm{DMSO})$ : $V(\mathrm{NMP})=1: 1: 1,22.5 \mathrm{~g} \mathrm{Ac-Glu(OtBu)-Glu(OtBu)-}$ 
Met-OH, 5.43 g HOBT, 12.85 g TBTU, 6.6 mL DIEA, 氮 气保护下室温反应, $0.05 \mathrm{~g} / \mathrm{mL}$ 狮三酮/乙醇液监测树脂 显色变化，约 $3.5 \mathrm{~h}$, 抽滤，分别用 $i-\operatorname{PrOH}(100 \mathrm{~mL} \times 2)$ 、 $V(\mathrm{DMF}): V(\mathrm{DMSO}): V(\mathrm{NMP})=1: 1: 1(100 \mathrm{~mL} \times 2)$ 和 $\mathrm{MeOH}(100 \mathrm{~mL} \times 3)$ 洗涤树脂, 抽滤干燥, 得到 Ac-Glu(OtBu)-Glu(OtBu)-Met-Gln(Trt)-Arg-Arg-MBHA Resin.

\subsection{3 合成 Ac-Glu-Glu-Met-Gln-Arg-Arg- $\mathrm{NH}_{2}$}

在装有 $\mathrm{Ac}-\mathrm{Glu}(\mathrm{OtBu})-\mathrm{Glu}(\mathrm{OtBu})-\mathrm{Met}-\mathrm{Gln}(\mathrm{Trt})-\mathrm{Arg}-$ Arg-MBHA Resin 的烧杯中加入 $60 \mathrm{~mL}$ 切割液 $V(\mathrm{TFA})$ : $V($ Phenol $): V($ 苯甲硫醚 $): V($ 水 $): V($ Tis $)=83: 5: 4$ ： $3: 5$, 室温搅拌 $2.5 \mathrm{~h}$, 抽滤, 将滤液加入 $300 \mathrm{~mL}$ 冷乙醚 中, 析出沉淀, 抽滤, 用冷乙醚淋洗固体 $(50 \mathrm{~mL} \times 2)$, 得 到六胜肽粗品 $7.6 \mathrm{~g}$, 经制备高效液相色谱纯化 $[\mathrm{A}(\mathrm{pH}$ $8.5 \mathrm{MeOH}), \mathrm{B}\left(\mathrm{pH} 8.5 \mathrm{H}_{2} \mathrm{O}\right), 5 \sim 45 \mathrm{~min}, 17 \% \sim 27 \%$ 梯度 洗脱], 收获纯品 $5.83 \mathrm{~g}$, 收率 65.58\%. m.p. 202.7 205.7 ${ }^{\circ} \mathrm{C}$, HPLC: A $(0.1 \%$ TFA/ACN $), \mathrm{B}\left(0.1 \% \mathrm{TFA} / \mathrm{H}_{2} \mathrm{O}\right), 25$ $\min 5 \% \sim 65 \%, 7.85 \mathrm{~min}$. 纯度 $98.38 \%,[\alpha]_{\mathrm{D}}^{20.5}-43.74(c$ $\left.0.88, \mathrm{CH}_{3} \mathrm{OH}\right) ;{ }^{1} \mathrm{H}$ NMR $\left(400 \mathrm{MHz}, \mathrm{D}_{2} \mathrm{O}\right) \delta: 4.38 \sim 4.30$ (m, 1H), $4.29 \sim 4.18(\mathrm{~m}, 3 \mathrm{H}), 4.14(\mathrm{dd}, J=14.2,8.1 \mathrm{~Hz}$, $2 \mathrm{H}), 3.16(\mathrm{~s}, 4 \mathrm{H}), 2.58(\mathrm{dd}, J=13.1,6.7 \mathrm{~Hz}, 1 \mathrm{H}), 2.54 \sim$ $2.45(\mathrm{~m}, 1 \mathrm{H}), 2.38 \sim 2.29(\mathrm{~m}, 2 \mathrm{H}), 2.21(\mathrm{dd}, J=14.9,7.5$ $\mathrm{Hz}, 4 \mathrm{H}), 2.07$ (s, 1H), 2.05 (s, 4H), 2.03 (s, 1H), 2.00 (s, $4 \mathrm{H}), 1.98 \sim 1.92(\mathrm{~m}, 3 \mathrm{H}), 1.92-1.86(\mathrm{~m}, 1 \mathrm{H}), 1.82(\mathrm{~s}, 2 \mathrm{H})$, 1.75 (s, 2H), $1.61(\mathrm{~d}, J=6.0 \mathrm{~Hz}, 4 \mathrm{H})$; IR (KBr) $v: 1710$, $1270,3270,1650,1410,3170,1540 \mathrm{~cm}^{-1}$; ESI-MS $m / z$ : $889.5\left(\mathrm{M}^{+}, 100\right), 890.45\left[\left(\mathrm{M}^{+}+1\right), 50\right], 445\left[\left(\mathrm{M}^{+}+1\right) / 2\right.$, 40], $701.9(30)$.

\section{References}

[1] Merrifield, R. B. J. Am. Chem. Soc. 1936, 85, 2149

[2] Bayer, E.; Mutter, M. Nature 1972, 237, 512.

[3] Ovchinnikov, M. V.; Bespalova, Z. D.; Molokoedov, A. S.; Revenko, I. V.; Sepetov, N. F.; Isakova, O. L.; Titov, M. I. Collect. Czech. Chem. Commun. 1989, 54, 772; 1989, 54, 784.

[4] (a) Gutiérrez, L. M.; Cànaves, J. M.; Ferrer-Montiel, A. V.; Reig, J. A.; Montal, M.; Viniegra, S. FEBS Lett. 1995, 372, 39.

(b) Ferrer-Montiel, A.V.; Gutiérrez, L. M.; Apland, J. P.; Canaves, J. M.; Gil, A.; Viniegra, S.; Biser, J.A.; Adler, M.; Montal, M. FEBS Lett. 1998, 435, 84.

(c) Blanes-Mira, C.; Merino, J. M.; Valera, E.; Fernández-Ballester, G.; Gutiérrez, L. M.; Viniegra, S.; Pérez-Payá, E.; Ferrer-Montiel, A. J. Neurochem. 2004, 88, 124.

[5] (a) Lungu, C.; Considine, E.; Zahir, S.; Ponsati, B.; Arrastia. S.; Hallett, M. Eur. J. Neurol. 2013, 20, 515.

(b) Fields, K.; Falla, T. J.; Rodan, K.; Bush, L. J. Cosmet., Dermatol. 2009, 8,8 .

(c) Lijuan, Z.; Falla, T. J. Clin. Dermatol. 2009, 27, 485.

[6] (a) Wang, Y.; Wang, M.; Xiao, X. S.; Huo, J.; Zhang, W. D. J. Cosmet. Laser Ther. 2013, 15, 237.

(b) Wang, Y.; Wang, M.; Xiao, S.; Pan, P.; Li, P.; Huo, J. Am. J. Clin. Dermatol. 2013, 14, 147.

[7] Blanes-Mira, C.; Clemente, J.; Jodas, G.; Gil, A.; Fernández-Ballester, G.; Ponsati, B.; Gutierrez, L.; Pérez-Payá, E.; Ferrer-Montiel, A. Int. J. Cosmet. Sci. 2002, 24, 303.

[8] (a) Jencks, W. P.; Carriuolo, J. J. Am. Chem. Soc. 1960, 82, 675. (b) Jencks, W. P.; Gilchrist, M. J. Am. Chem. Soc. 1966, 88, 104.

[9] Zhu, L.-L.; Sheng, Z.-C.; Chen, Y.-W.; Shen, S.-B. J. Chem. Eng. Chin. Univ. 2013, 27, 791 (in Chinese) (朱亮亮, 绳则翠, 陈英文, 沈树宝, 高校化学工程学报, 2013, 27, 791.)

[10] Mutter, M.; Maser, F. In Peptides 1982, Ed.: Blaha, K., Berlin, 1983, p. 729

[11] Han, X.; Wang, D. X. Acta Pharm. Sin. 2007, 42, 111 (in Chinese). (韩香, 王德心, 药学学报, 2007, 42, 111.) 